\title{
Performance Analysis of Spatially Distributed MIMO Systems
}

\author{
Farhana Bashar* and Thushara D. Abhayapala \\ Research School of Engineering, Australian National University, Canberra 0200 ACT, Australia \\ *farhana.bashar@anu.edu.au
}

\begin{abstract}
With the growing popularity of ad-hoc sensor networks, spatially distributed MIMO systems have drawn a lot of attention. This work considers a spatially distributed MIMO system with randomly distributed transmit and receive antennas over spatial regions. We use the modal decomposition of wave propagation to analyse the performance limits of such system, since the sampling of the spatial regions populated with antennas is a form of mode excitation. Specifically, we decompose signals into orthogonal spatial modes and apply concepts of MIMO communications to quantify the instantaneous capacity and the outage probability. Our analysis shows that analogous to conventional point-to-point MIMO system, the instantaneous capacity of spatially distributed MIMO system over Rayleigh fading channel is equivalent to a Gaussian random variable. Afterwards, we derive an accurate closed-form expression for the outage probability of proposed system utilizing the definition of instantaneous capacity. Besides, in rich scattering environment, the spatially distributed MIMO system provides best performance when the spatial regions are of same size, and each region is equipped with equal number of antennas. Furthermore, to facilitate the total transmit power allocation among the channels, we propose an algorithm which indicates a significant performance improvement over conventional equal transmit power allocation scheme, even at low SNR.
\end{abstract}

\section{Introduction}

Recently, there has been a great deal of interest in spatially distributed multiple-input multipleoutput (MIMO) systems where transmit and receive antennas are distributed randomly in spatial regions [1-4]. Such systems can deliver all the attractive benefits of conventional MIMO in the point-to-point wireless channel, but at a much larger scale. More precisely, spatially distributed MIMO systems can provide large performance gains, since realistic capacity limits are best explained by considering the antennas over finite spatial diversity [5-10]. In a distributed setting, antennas are typically much farther apart from each other as compared to conventional MIMO. Thus, one of the key assumptions exploited by spatially distributed MIMO is that the channels between different pairs of antennas are independent. In consequence, analogous to the point-to-point case, it seems appropriate to achieve capacity gains linear to the number of antennas contained in transmit and receive regions. As a result, several works [11-15] studied the instantaneous capacity and the outage probability of spatially distributed MIMO systems subject to antenna elements.

However, antenna elements are not the limiting factor in the performance analysis of multiantenna systems. They are simply one choice of implementation of a general spatial processing underlying all wireless communication systems. It was demonstrated in [16] that the sampling of the spatial regions populated with antennas is a form of mode excitation with each mode of 
the transmit region coupling to a mode of the receive region via the scattering environment. This work $^{1}$ aims to analyse the instantaneous capacity and the outage probability of spatially distributed MIMO systems from a mode-to-mode communication perspective.

This work considers a spatially distributed MIMO system between arbitrary shaped spatial regions with each region containing antennas distributed at random regardless of the number of antennas or array geometry. We develop a novel framework to represent communication between the regions in terms of orthonormal basis functions encoded in wave modes. This modal representation describes the outgoing/ incoming signals in the transmit/ receive regions in terms of transmit/ receive modes in non-line of sight, rich scattering environments. This mathematical framework is similarly used in [16] and [18-20], respectively, to evaluate the intrinsic capacity of continuous space channels and the best-connected communication channels between arbitrary shaped regions. In this work, we use the formalism of $[16,18-20]$ as the foundation to analyse the instantaneous capacity and the outage probability of spatially distributed MIMO systems for narrowband signal transmissions subject to Rayleigh fading.

Some specific contributions made in this work are:

1. It is well established in literature that the mutual information (MI), also known as instantaneous capacity, of conventional MIMO system over Rayleigh fading channel is known to be equivalent to a Gaussian random variable [21-24]. As far as distributed MIMO system is concerned, its instantaneous capacity has a similar formulation as the instantaneous capacity of conventional MIMO system over the Rayleigh fading channel [25]. In this paper, we first show that the instantaneous capacity of proposed distributed MIMO system for modeto-mode communication is also equivalent to a Gaussian random variable. Based on this definition of instantaneous capacity, we then derive an accurate closed-form expression for the outage probability of the proposed distributed MIMO system.

2. Analogous to conventional MIMO systems, the distributed MIMO systems provide best performance with each spatial region containing equal number of randomly distributed antennas, populating regions with different number of antennas simply adds redundancy to the system. However, since the number of effective antennas is dependent on size of the region, to obtain the best performance, we need to consider equal sized regions.

3. To facilitate the total transmit power allocation among the effective independent channels, we look at a descending transmit power allocation algorithm. Our proposed algorithm depends on how the total transmit power is distributed in a descending order among the channels. The simulation results indicate that for a uniform descending order power allocation among the independent channels, the proposed power allocation algorithm outperforms the conventional equal power allocation scheme at both low and high average SNR. On the contrary, allocating most of the transmit power to the most favorable channel, the proposed algorithm provides better performance compared to the conventional scheme at low average SNR. In addition, a desired spectral efficiency is attainable (at both low and high average SNR) with a higher probability by employing the proposed algorithm than applying the equal power allocation scheme. This outcome is feasible irrespective of how the total transmit power is allocated in a descending order among the independent channels. However, for a desired spectral efficiency, allocating most of the transmit power to the most favorable channel provides large performance gains compared to uniform descending order power allocation among channels.

\footnotetext{
${ }^{1}$ A preliminary version of this work has been accepted at ICSPCS 2016 [17]. However, this journal version has significant contribution to the conception, framework and interpretation of the reported study.
} 


\section{Communication Between Spatial Regions}

We consider a spatially distributed MIMO transmission between arbitrary shaped spatial regions where each region is populated with randomly distributed antennas. The system schematic is shown in Fig. 1.

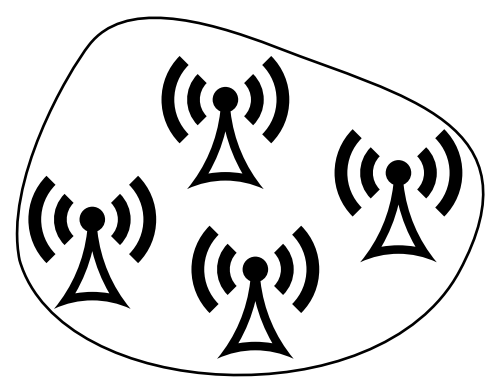

Transmit Region

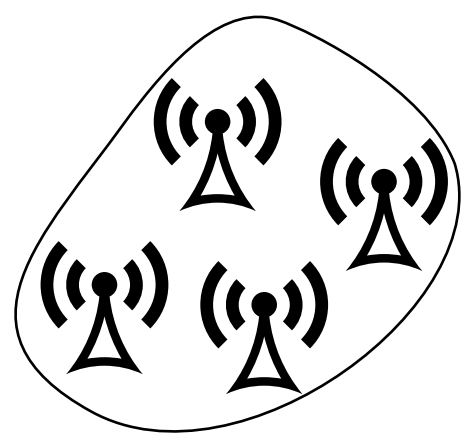

Receive Region

Fig. 1. Spatially distributed MIMO system schematic.

Assume that the transmit region, denoted by $\Omega_{T}$, is excited by some means that radiates signals into the surrounding complex scattering environment and after propagation via the complex scattering environment the transmitted signals arrive at the receive region, denoted by $\Omega_{R}$. Denote $\Phi(\hat{\phi})$ as the source signal radiating in direction $\hat{\phi}$ from the surface of the transmit region $\Omega_{T}$. Let $\Psi(\hat{\psi})$ denote the signal received from direction $\hat{\psi}$ to the receive region $\Omega_{R}$, generated by the transmitted signals, given by

$$
\Psi(\hat{\boldsymbol{\psi}})=\int_{\Omega_{T}} g(\hat{\boldsymbol{\phi}}, \hat{\boldsymbol{\psi}}) \Phi(\hat{\boldsymbol{\phi}}) d s(\hat{\boldsymbol{\phi}})
$$

where $d s(\hat{\phi})$ is a surface element of transmit region $\Omega_{T}$ with unit normal $\hat{\phi}$ and $g(\hat{\phi}, \hat{\psi})$ is the effective complex random scattering gain function of the scattering environment for signals radiating from the transmit region in direction $\hat{\phi}$ and arriving at the receive region along $\hat{\psi}$. The spatial channel model for communication between the spatial regions in shown in Fig. 2 where all scatters are considered external to regions $\Omega_{T}$ and $\Omega_{R}$.

Note that the scattering gain function $g(\hat{\phi}, \hat{\boldsymbol{\psi}})$ describes the channel, given that the received signal is generated at $\hat{\psi} \in \Omega_{R}$ due to the input function $\Phi(\hat{\phi}), \hat{\phi} \in \Omega_{T}$. Furthermore, in the absence of scatterer the scattering gain function is given by [26]

$$
g(\hat{\boldsymbol{\phi}}, \hat{\boldsymbol{\psi}})=\frac{e^{-i k\|\hat{\boldsymbol{\phi}}-\hat{\boldsymbol{\psi}}\|}}{4 \pi\|\hat{\boldsymbol{\phi}}-\hat{\boldsymbol{\psi}}\|}
$$

which is the fundamental solution to the Helmholtz equation for a point source at $\hat{\phi}$ [27, p. 198]. Here, $k$ is the scalar wavenumber and $i=\sqrt{-1}$. However, scattering environments result into much more complicated expression for the channel $g(\hat{\phi}, \hat{\psi})$ than $(2)$.

\subsection{Basis Function Representation of Signals}

To describe the arbitrary outgoing source function $\Phi(\hat{\phi})$ in the transmit region $\Omega_{T}$, we choose a complete orthonormal basis set of functions that is defined within $\Omega_{T}$, donated as $\left\{\phi_{1}(\hat{\phi}), \phi_{2}(\hat{\phi}), \ldots\right\}$ 


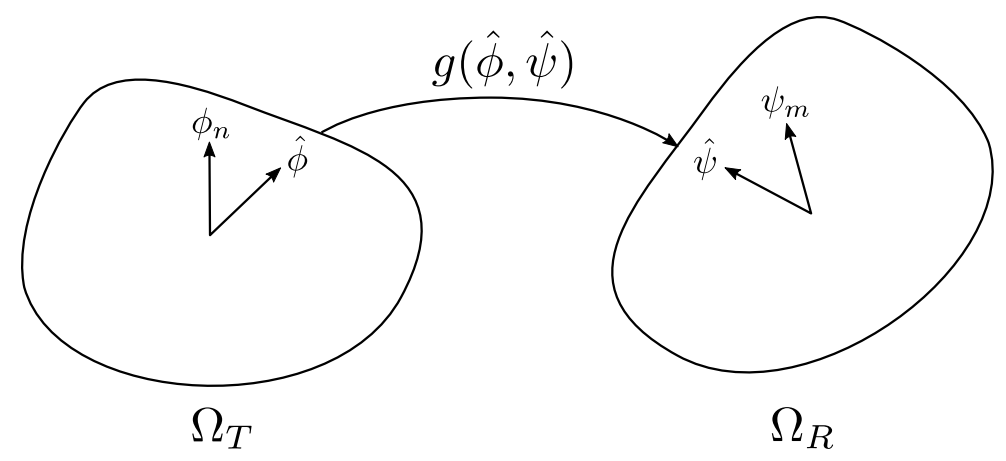

Fig. 2. Spatial channel model with $g(\hat{\phi}, \hat{\psi})$ representing the effective complex random scattering gain function of the scattering environment for signals radiating from the transmit region in direction $\hat{\phi}$ and arriving at the receive region along $\hat{\psi}$.

with the basis set satisfying the orthonormality relation

$$
\int_{\Omega_{T}} \phi_{n}(\hat{\phi}) \phi_{n^{\prime}}^{*}(\hat{\phi}) d s(\hat{\phi})=\delta\left(n-n^{\prime}\right)
$$

The source function $\Phi(\hat{\phi})$ can, therefore, be represented by the basis set as follows

$$
\Phi(\hat{\phi})=\sum_{n} s_{n} \phi_{n}(\hat{\phi})
$$

where $s_{n}$ are the series coefficients given by the projection of $\phi_{n}$ on $\Phi$;

$$
s_{n}=\left\langle\Phi(\hat{\phi}), \phi_{n}(\hat{\phi})\right\rangle_{\Omega_{T}} .
$$

Similarly, to describe the arbitrary incoming receive function $\Psi(\hat{\psi})$ in the receive region $\Omega_{R}$, we choose a complete orthonormal basis set of functions that is defined within $\Omega_{R}$, namely, $\left\{\psi_{1}(\hat{\boldsymbol{\psi}}), \psi_{2}(\hat{\boldsymbol{\psi}}), \ldots\right\}$ with the basis set satisfying the orthonormality relation

$$
\int_{\Omega_{R}} \psi_{m}(\hat{\boldsymbol{\psi}}) \psi_{m^{\prime}}^{*}(\hat{\boldsymbol{\psi}}) d s(\hat{\boldsymbol{\psi}})=\delta\left(m-m^{\prime}\right)
$$

Henceforth, the receive function $\Psi(\hat{\psi})$ can be represented by the basis set as follows

$$
\Psi(\hat{\boldsymbol{\psi}})=\sum_{m} y_{m} \psi_{m}(\hat{\boldsymbol{\psi}})
$$

where $y_{m}$ are the series coefficients given by the projection of $\psi_{m}$ on $\Psi$;

$$
y_{m}=\left\langle\Psi(\hat{\boldsymbol{\psi}}), \psi_{m}(\hat{\boldsymbol{\psi}})\right\rangle_{\Omega_{R}}
$$

It should be noted that in the context of conventional MIMO systems, works of [28-31] suggested similar mathematical structures. To determine the capacity of any spectrally shaped MIMO channel, these works applied vector-coding schemes by decomposing the channel into infinite set of parallel channels using a complete orthonormal basis set of continuous time, frequency and/or space functions. 
Now, observe that the coefficient $s_{n}$ (5) corresponds to the transmitted signal encoded in the $n^{\text {th }}$ mode of the expression (4). Similarly, $y_{m}$ (8) corresponds to the received signal encoded in the $m^{\text {th }}$ mode of the expression (7). To compute the information content of the continuous spatial channel, we need to develop the relationship between the transmit and receive modes. Consider that the received signal from the direction $\hat{\psi}$ due to the transmitted signal $s_{n}$ encoded in the $n^{\text {th }}$ transmit mode is given by

$$
\Psi_{n}(\hat{\boldsymbol{\psi}})=s_{n} \int_{\Omega_{T}} g(\hat{\boldsymbol{\phi}}, \hat{\boldsymbol{\psi}}) \phi_{n}(\hat{\boldsymbol{\phi}}) d s(\hat{\boldsymbol{\phi}})
$$

The total received signal can, thus, be evaluated by taking the sum over all the transmit modes as follows

$$
\Psi(\hat{\psi})=\sum_{n} \Psi_{n}(\hat{\psi})
$$

Observe that from (8), (9) and (10), the relationship between the $m^{\text {th }}$ mode received signal $y_{m}$ due to the transmitted signals encoded in the $n$ modes is given by

$$
\begin{aligned}
y_{m} & =\sum_{n}\left\langle\Psi_{n}(\hat{\boldsymbol{\psi}}), \psi_{m}(\hat{\boldsymbol{\psi}})\right\rangle_{\Omega_{R}} \\
& =\sum_{n} \beta_{m}^{n} s_{n}
\end{aligned}
$$

where

$$
\begin{aligned}
\beta_{m}^{n} & =\left\langle\left\langle g(\hat{\boldsymbol{\phi}}, \hat{\boldsymbol{\psi}}), \phi_{n}^{*}(\hat{\boldsymbol{\phi}})\right\rangle_{\Omega_{T}}, \psi_{m}(\hat{\boldsymbol{\psi}})\right\rangle_{\Omega_{R}} \\
& =\int_{\Omega_{R}} \int_{\Omega_{T}} g(\hat{\boldsymbol{\phi}}, \hat{\boldsymbol{\psi}}) \phi_{n}(\hat{\boldsymbol{\phi}}) \psi_{m}^{*}(\hat{\boldsymbol{\psi}}) d s(\hat{\boldsymbol{\phi}}) d s(\hat{\boldsymbol{\psi}})
\end{aligned}
$$

The variables $\beta_{m}^{n}$ can be thought of as the coupling coefficients between the $n^{\text {th }}$ transmit mode in region $\Omega_{T}$ and the $m^{t h}$ receive mode in region $\Omega_{R}$.

Note that (11) via the representations (4) and (7) is an exactly equivalent to (1). Instead of expressing the value of the signal at a given point in $\Omega_{R}$ directly, we have given the coefficients $y_{m}$ for the basis functions $\psi_{m}(\hat{\psi})$. It can be conceptually useful to think of (11) in matrix terms, in which case we can rewrite it as

$$
\left[\begin{array}{c}
y_{1} \\
y_{2} \\
y_{3} \\
\cdot \\
\cdot \\
\cdot
\end{array}\right]=\left[\begin{array}{ccccc}
\beta_{1}^{1} & \beta_{1}^{2} & \cdot & \cdot & \cdot \\
\beta_{2}^{1} & \beta_{2}^{2} & \cdot & \cdot & \cdot \\
\beta_{3}^{1} & \beta_{3}^{2} & \cdot & \cdot & \cdot \\
\cdot & \cdot & \cdot & \cdot & \cdot \\
\cdot & \cdot & \cdot & \cdot & \cdot \\
\cdot & \cdot & \cdot & \cdot & \cdot
\end{array}\right]\left[\begin{array}{c}
s_{1} \\
s_{2} \\
s_{3} \\
\cdot \\
\cdot \\
\cdot
\end{array}\right]
$$

where it should be noted that these matrices will be infinite.

\subsection{Communication Modes}

So far, we have used basis sets in the regions $\Omega_{T}$ and $\Omega_{R}$ encoded in infinite number of modes. We can reasonably ask whether there is a finite limit to the number of modes to be considered for which the mathematical results become particularly simple and the physical interpretation becomes 
clearer. In fact, for any pair of regions, it is possible to determine such limits to the number of modes, and we can view these as defining communications modes. In this section, we proceed to derive these finite dimensional sets.

We start with utilizing the formalism of the previous section to derive a sum rule on the strengths of the coupling coefficients. The work of [19] provided a sum rule on the strengths of the coupling coefficients for a point source at any particular position considering scatter-free environment. In contrast, the sum rule derived in this section is more general, since we consider random scattering.

The sum rule is derived by expansion of the complex scattering gain function $g(\hat{\phi}, \hat{\psi})$ in the orthonormal basis sets. Note that based on (12), the Fourier series expansion of $g(\hat{\phi}, \hat{\psi})$ is given by

$$
g(\hat{\boldsymbol{\phi}}, \hat{\boldsymbol{\psi}})=\sum_{n} \sum_{m} \beta_{m}^{n} \phi_{n}^{*}(\hat{\boldsymbol{\phi}}) \psi_{m}(\hat{\boldsymbol{\psi}})
$$

Then we have

$$
|g(\hat{\boldsymbol{\phi}}, \hat{\boldsymbol{\psi}})|^{2}=\left[\sum_{n} \sum_{m} \beta_{m}^{n} \phi_{n}^{*}(\hat{\boldsymbol{\phi}}) \psi_{m}(\hat{\boldsymbol{\psi}})\right]\left[\sum_{n} \sum_{m}\left(\beta_{m}^{n}\right)^{*} \phi_{n}(\hat{\boldsymbol{\phi}}) \psi_{m}(\hat{\boldsymbol{\psi}})^{*}\right] .
$$

Now, integrating both sides of the above equation over the regions $\Omega_{T}$ and $\Omega_{R}$ and using the orthonormality relations of (3) and (6) of the basis sets, we obtain

$$
G(\hat{\phi}, \hat{\psi})=\sum_{n} \sum_{m}\left|\beta_{m}^{n}\right|^{2}
$$

where

$$
G(\hat{\boldsymbol{\phi}}, \hat{\boldsymbol{\psi}})=\int_{\Omega_{R}} \int_{\Omega_{T}}|g(\hat{\boldsymbol{\phi}}, \hat{\boldsymbol{\psi}})|^{2} d s(\hat{\boldsymbol{\phi}}) d s(\hat{\boldsymbol{\psi}})
$$

represents the total connection strength over departure and arrival angles $\phi$ and $\psi$, respectively, with normalization

$$
\int_{\Omega_{R}} \int_{\Omega_{T}} G(\hat{\phi}, \hat{\boldsymbol{\psi}}) d s(\hat{\phi}) d s(\hat{\boldsymbol{\psi}})=1
$$

Thus, the total connection strength $G(\hat{\phi}, \hat{\psi})$ is computed by the evaluation of the complex scattering gain function $g(\hat{\boldsymbol{\phi}}, \hat{\boldsymbol{\psi}})$ over the spatial regions $\Omega_{T}$ and $\Omega_{R}$. In effect, (16) indicates that the sum of the modulus squared of the coupling coefficients depend only on the shapes of the regions and the scattering environment, not on the choice of basis sets in each region. Rather, as long as the basis sets are complete and orthonormal, this result is true independent of the basis sets we choose in each region. Therefore, only the geometry of the regions and the scattering environment will determine the possible limits to communication between the regions.

Furthermore, it is clear from (16) that there is some maximum value of $\left|\beta_{m}^{n}\right|^{2}$. Hence, there must be some pair $\left[\phi_{1}(\hat{\phi}), \psi_{1}(\hat{\psi})\right]$ of source and receive functions that are most strongly coupled, i.e., a pair for which the coupling coefficient $\left|\beta_{1}^{1}\right|$ has the largest squared modulus $\left|\beta_{1}^{1}\right|^{2}$. We can now find the second members $\left[\phi_{2}(\hat{\phi}), \psi_{2}(\hat{\psi})\right]$ of the basis sets, such that these functions are orthogonal to the corresponding first members. The corresponding coupling coefficient $\left|\beta_{2}^{2}\right|$ will have the nextlargest squared modulus value. We can continue to proceed in this manner to find all the other 
members of the basis set, requiring that each successive member of each set be orthogonal to all the previous members of that set.

Note that the integral on the right hand side of (17) is finite, stating that the total connection strength $G(\hat{\phi}, \hat{\psi})$ is bounded. Then according to (16), the total strength of coupling between the regions is bounded. Further, since the members of the basis set are chosen in such a way that $\left|\beta_{1}^{1}\right|^{2}>\left|\beta_{2}^{2}\right|^{2}>\cdots>\left|\beta_{m}^{n}\right|^{2}$, the higher order modes will lead to vanishingly small basis function components (vanishingly small $\left|\beta_{m}^{n}\right|$ ). In effect, the number of basis function pairs must be finite and $\left|\beta_{m}^{n}\right|$ is negligible for all but a finite set of modes $^{2}$.

\subsection{Mode-to-Mode Communication}

In this section, we utilize the findings of the previous sections to develop a framework for distributed MIMO systems from the mode-to-mode communication perspective.

Based on the discussion of the previous section, form a communication point of view, an infinite number of modes are excited for each spatial region irrespective of the number or location of the transmit/ receive antennas. However, there is a very little energy associated with the higher order modes. In terms of practical systems, these weakly connected modes lead to weak receiving waves that become insignificant when the presence of noise is considered. In effect, the infinite dimensional representation (11) can be truncated to a finite set of appropriately chosen transmit modes (cardinality $N$ ) and receive modes (cardinality $M$ ), such that

$$
\left|\beta_{m}^{n}\right| \leq \epsilon, \quad \text { for } n>N, m>M \text { and small } \epsilon \text {. }
$$

In consequence, consider that the transmitted signals are denoted by a $N \times 1$ vector $s$ where $\left\{s_{n}\right\}_{n=1}^{N}$ refers to the $n^{\text {th }}$ transmit mode signal. The total power of the transmitted signal $s$ is constrained to $P_{T}$ regardless of the number of transmit modes. In this work, we assume that the total transmit power $P_{T}$ is assigned to the effective modes at the scatter-free transmit region in such a way that

$$
\operatorname{tr}\{\boldsymbol{Q}\} \leq P_{T}
$$

where $\boldsymbol{Q}=E\left\{\boldsymbol{s} \boldsymbol{s}^{\dagger}\right\}$ is the covariance of $\boldsymbol{s}$ and non-negative define $(\boldsymbol{Q} \geq 0)$. Here, $E\{\cdot\}$ represents the expectation operator and $s^{\dagger}$ is the complex conjugate transpose of $s$.

Furthermore, we consider that the transmitted signal bandwidth is narrow enough, thus, its frequency response can be considered as flat. Then the signals received at the receive region is given by

$$
\boldsymbol{y}=\boldsymbol{H} s+\boldsymbol{z}
$$

where $\boldsymbol{y}$ is an $M \times 1$ vector denoting the $M$ receive mode signals $\left\{y_{m}\right\}_{m=1}^{M}, \boldsymbol{z}$ is an $M \times 1$ noise vector and $\boldsymbol{H}$ is the $M \times N$ scattering channel matrix.

Note that the $(m, n)^{t h}$ element of the channel matrix $\boldsymbol{H}$ is given by (12), such that

$$
\boldsymbol{H}=\left[\begin{array}{cccc}
\beta_{1}^{1} & \beta_{1}^{2} & \ldots & \beta_{1}^{N} \\
\beta_{2}^{1} & \beta_{2}^{2} & \ldots & \beta_{2}^{N} \\
\vdots & \vdots & \ddots & \vdots \\
\beta_{M}^{1} & \beta_{M}^{2} & \ldots & \beta_{M}^{N}
\end{array}\right]
$$

\footnotetext{
${ }^{2}$ A detailed discussion of the this formulation is available in [19].
} 
The $(m, n)^{t h}$ entry $\beta_{m}^{n}$ of the matrix $\boldsymbol{H}$ represents the channel fading coefficient (gain) from the $n^{\text {th }}$ transmit mode to $m^{\text {th }}$ receive mode.

In addition, this work considers additive Gaussian noise which is assumed to be white and has a given spectral density function. Hence, the components of $z$ are statistically independent complex zero mean Gaussian random variables with covariance

$$
E\left\{\boldsymbol{z} \boldsymbol{z}^{\dagger}\right\}=\sigma^{2} \boldsymbol{I}_{M}
$$

where $\sigma^{2}$ is the identical noise power per receive mode and $\boldsymbol{I}_{M}$ is the $M \times M$ identity matrix.

Therefore, the average signal to noise ratio (SNR) at each receive mode is defined as

$$
\rho=\frac{P_{T}}{\sigma^{2}} .
$$

Moreover, we consider non-line of sight propagation and a zero-mean uncorrelated scattering environment $\left(\right.$ Rayleigh $\left.^{3}\right)$.

Based on the formalism of this section, the following section evaluates the performance limits of spatially distributed MIMO systems for narrowband signal transmissions.

\section{Capacity Limits of Spatially Distributed MIMO Systems}

Up to this point, we have developed a narrowband spatially distributed MIMO system between arbitrarily shaped spatial regions. The proposed system takes a power constrained function as its input and has a function as its output. The output is then corrupted by additive white Gaussian noise. Now, we can proceed to use this formalism to evaluate the instantaneous capacity and outage capacity probability of the system. Before proceeding to the main results presented in this work, we first determine the number of independent channels available between the spatial regions.

\subsection{Favourable Propagation Characteristics}

Independent channels between transmit and receive region is one of the most important characteristics of the favourable propagation in spatially distributed MIMO systems subject to Rayleigh fading. Although there are infinitely many connections between the regions, finite number of independent practical channels are available to carry information between transmit and receive regions.

In conventional MIMO systems the rank of the channel matrix determines the maximum number of independent parallel channels between the transmitter array and the receiver array. In the literature [33], the idealistic model assumes that the channel matrix has independent elements corresponding to sufficiently spaced antennas. However, when realistic antenna configurations and scattering environments are considered, the elements of the channel matrix become correlated, and the number of possible independent parallel channels is reduced.

In corollary, in case of spatially distributed MIMO communications, the rank of the scattering channel matrix $\boldsymbol{H}$ (22) would determine the maximum number of independent parallel channels between the transmit and the receive regions. Therefore, number of independent parallel channels

$$
K=\operatorname{rank}(\boldsymbol{H}) \leq \min \{N, M\}
$$

\footnotetext{
${ }^{3}$ Rayleigh fading model represents excellent approximation of practical scenarios as it describes the effect of heavily built-up urban environments on radio signals [32].
} 
with the richness factor

$$
k_{s}=\frac{\operatorname{rank}(\boldsymbol{H})}{\min \{N, M\}}
$$

where $k_{s} \in(0,1]$ characterizes the scattering environment, such that

1. $k_{s}=1$ corresponds to a uncorrelated high rank channel model where the elements of the channel matrix $H$ are independent. Thus, characterizes a rich scattering environment.

2. $k_{s}<1$ corresponds to a correlated low rank channel model. Therefore, the scattering environment is not rich enough to support the full set of independent modal gains.

\subsection{Capacity Limits}

We are now in position to derive exact closed-form expressions for the instantaneous capacity and outage probability of spatially distributed MIMO systems for mode-to-mode communications. We start with evaluating the instantaneous capacity of spatially distributed MIMO systems for narrowband transmissions.

\section{Instantaneous Capacity}

The instantaneous capacity of the spatially distributed MIMO system can simply be characterized in terms of the mutual information between the transmit and receive regions.

Hence, based on the characteristics of system model (21), we can define the instantaneous capacity as [34]

$$
\begin{aligned}
C_{\text {ins }} & =\mathcal{I}(\boldsymbol{s}, \boldsymbol{y} \mid \boldsymbol{H}) \\
& =\log \left|\boldsymbol{I}_{M}+\frac{1}{\sigma^{2}} \boldsymbol{H} \boldsymbol{Q} \boldsymbol{H}^{\dagger}\right| \\
& =\log \left|\boldsymbol{I}_{N}+\frac{1}{\sigma^{2}} \boldsymbol{Q} \boldsymbol{H}^{\dagger} \boldsymbol{H}\right|
\end{aligned}
$$

where $\boldsymbol{I}_{M}$ and $\sigma^{2}$ are defined in (23) and $\boldsymbol{Q}$ is defined in (20). Further, $\boldsymbol{I}_{N}$ is the $N \times N$ identity matrix. Note that (27) follows from the determinant identity $|\boldsymbol{I}+\boldsymbol{A} \boldsymbol{B}|=|\boldsymbol{I}+\boldsymbol{B} \boldsymbol{A}|$.

However, the singular value decomposition (SVD) theorem or eigen channel based evaluation of conventional MIMO instantaneous capacity is well established in literature [35-42]. Utilizing (27), the following theorem provides the SVD theorem or eigen channel based instantaneous capacity of spatially distributed MIMO systems.

Theorem 1. For a given constraint on the total transmit power $P_{T}$, the instantaneous capacity of any spatially distributed MIMO system for mode-to-mode communication over Rayleigh fading channels is given by

$$
C_{\text {ins }}=\sum_{k=1}^{K} \log \left(1+\frac{1}{\sigma^{2}} P_{k} \lambda_{k}\right) .
$$

where $K=\min \{N, M\}$ represents the number of independent channels with $N$ and $M$ denoting the number of transmit and receive modes, $\sigma^{2}$ is the identical additive white Gaussian noise (AWGN) power per receive mode, $P_{k}$ is the power of the $k^{t h}$ channel such that $\sum_{k=1}^{K} P_{k} \leq P_{T}$ and $\lambda_{k}$ is the $k^{t h}$ eigenvalue of the channel matrix. 
Proof of the theorem is given in Appendix 1.

It is clear from the theorem that analogous to conventional MIMO systems [21-24], for modeto-mode communications between spatial regions, the instantaneous capacity of any spatially distributed MIMO system over Rayleigh fading channel is equivalent to a Gaussian random variable.

Next, we proceed to quantify the outage capacity probability of narrowband spatially distributed MIMO systems.

\section{Outage Capacity Probability}

By definition, the outage capacity probability, denoted by $P_{\text {out }}$ is the probability of not achieving a threshold capacity, outage capacity.

Thus, the outage probability for spectral efficiency $R$ is defined as

$$
P_{\text {out }}=\operatorname{Pr}\{\mathcal{I}(\boldsymbol{s}, \boldsymbol{y} \mid \boldsymbol{H})<R\}
$$

where $\mathcal{I}(\boldsymbol{s}, \boldsymbol{y} \mid \boldsymbol{H})=C_{\text {ins }}$ is defined in Theorem 1. Now, based on this definition we provide the following theorem to determine the outage probability of narrowband spatially distributed MIMO systems.

Theorem 2. Considering narrowband signal transmissions between arbitrary shaped spatial regions in terms of orthonormal basis functions encoded in wave modes to describe the outgoing/ incoming signals in transmit/ receive region, outage probability of any spatially distributed MIMO system subject to Rayleigh fading is

$$
P_{\text {out }}=\prod_{k=1}^{K}\left(1-e^{-2^{R} \zeta_{k}}\right), \quad 2^{R} \geq 0
$$

where $R$ is the desired spectral efficiency and

$$
\zeta_{k}=\frac{1}{1+\lambda_{k} P_{k} / \sigma^{2}}
$$

is the rate parameter of Rayleigh distribution. In addition, $K, \sigma^{2}, P_{k}$ and $\lambda_{k}$ are defined in Theorem 1.

A comprehensive proof of the theorem is given in Appendix 2.

In the following section, we elucidate some physical insights of the derived theorems.

\subsection{Discussion}

Observe that Theorem 1 and Theorem 2 represent closed-form expressions for the instantaneous capacity and the outage probability of narrowband spatially distributed MIMO systems. These expressions indicate that the available mutual information in the spatially distributed MIMO system, as well as the distributed MIMO outage probability, is limited by the number of independent channels available between the transmit and the receive regions. Note that as discussed in Section 3.1, the rank of the channel matrix $\boldsymbol{H}$ determines the effective number of independent parallel channels between the transmit and receive regions. Further, the effective number of independent channels $K$, as shown in (25), is dependent on the effective number of modes excited in the regions.

In this context, for any arbitrary antenna configuration (number of antennas and their location), $[7,43,44]$ developed a modal decomposition method that maps the transmitted signals to 
the transmit modes and the receive modes to received signals for circular (and/or spherical) transmit and receive regions. The modal decomposition method showed that the effective number of modes excited in the transmit and the receive regions are limited by the size of the regions. For instance, if $r_{T}$ and $r_{R}$ denote the radius of the circular transmit and receive regions, respectively, then the effective number of modes become $N=2 \mathcal{N}+1$ and $M=2 \mathcal{M}+1$, where $\mathcal{N}=\left\lceil e \pi r_{T} / \lambda\right\rceil$ and $\mathcal{M}=\left\lceil e \pi r_{R} / \lambda\right\rceil$. Here, $\lambda$ represents the wavelength corresponding to the operating frequency. Furthermore, for spherical transmit and receive regions, the effective number of modes are, respectively, $N=(\mathcal{N}+1)^{2}$ and $M=(\mathcal{M}+1)^{2}$.

As discussed in Section 3.1, a rich scattering environment is capable of providing the maximum number of independent links between transmitter modes to receiver modes. Therefore, the channel matrix $\boldsymbol{H}$ of a rich environment has the greatest rank of $\min \{2 \mathcal{N}+1,2 \mathcal{M}+1\}$ for circular transmit and receive regions (or $\min \left\{(\mathcal{N}+1)^{2},(\mathcal{M}+1)^{2}\right\}$ for spherical regions). In effect, in a rich scattering environment, the maximum outcomes from (28) and (30) are achieved when equal number of transmit and receive modes are excited. In other words, the best performance requires equal sized regions. However, if the scattering media is not rich, i.e., not enough reflectors/scatteres such as trees, buildings etc., then the rank of $\boldsymbol{H}$ could be less than $\min \{2 \mathcal{N}+1,2 \mathcal{M}+1\}$ for circular transmit and receive regions (or $\min \left\{(\mathcal{N}+1)^{2},(\mathcal{M}+1)^{2}\right\}$ for spherical regions).

Furthermore, We can interpret the mode to mode communication between the transmit and receive regions in the context of antenna elements. Consider that $n_{T}$ and $n_{R}$ antennas are located inside the transmit and receive regions of radius of radius $r_{T}$ and $r_{R}$, respectively. The work of [45] showed that we can regard up to $n_{T}-N$ of transmit antennas and $n_{R}-M$ of receive antennas as superfluous, that is, any number of antennas in the transmit (or receive) region beyond the effective number of transmit (or receive) modes $N$ (or $M$ ), provide little or no additional information, depending on how wisely the antennas are spaced. This guides how densely we can usefully populate space with antennas. For small circular (or spherical) transmit and receive regions, the effective number of transmit and receive modes is small, and the asymptotic analysis and predictions about performance enhancements should be cautiously interpreted.

Based on the above discussion, it is evident that in a rich scattering environment, the narrowband spatially distributed MIMO systems provide best performances with each spatial region containing equal number of randomly distributed antennas. Populating regions with different number of antennas simply adds redundancy to the system. However, It remains an open problem to determine the best placement of a given number of transmit and receive antenna elements in circular (or spherical) regions to maximize the performance limits or minimize other system-related cost functions.

In addition, in conventional MIMO systems antennas can be easily synchronized, which is critical to the performance of the systems. In case of the spatially distributed MIMO systems, it is not practically feasible to synchronize significantly spaced antennas in the transmit (or receive) region. However, for mathematical simplicity, we take the most liberal, permissive attitude towards cooperation, i.e., synchronization and full coordination among the antennas in the transmit region as well as among the antennas in the receive region.

\section{Transmit Power Allocation}

This section discusses the transmit power allocation schemes to distribute the total transmit power among the independent channels between the circular (or spherical) transmit and receive regions. We begin with considering that the channel matrix is deterministic, i.e. the receive region is aware 
of the realization of the channel, while the transmit region only knows the statistics of the channel.

In this context, the pioneering works of $[34,46]$ showed that in conventional MIMO systems, when the receiver is aware of the realization of the channel, but the transmitter only knows the statistics of the channel, it is optimal to uniformly distribute the total transmit power equally among the available independent channels. Analogous to conventional MIMO systems, we study the equal transmit power allocation scheme for spatially distributed MIMO systems as follows.

\section{Equal Transmit Power Allocation:}

In this case, the total transmit power $P_{T}$ is distributed equally among the $K$ channels, hence, power at each channel is

$$
P_{k}=\frac{P_{T}}{K}
$$

Therefore, using the definition of the average SNR per receive mode $\rho(24)$, the instantaneous capacity (28) can be written as

$$
C_{\text {ins }}=\sum_{k=1}^{K} \log \left(1+\frac{\rho}{K} \lambda_{k}\right) .
$$

Further, (31) yields

$$
\zeta_{k}=\frac{1}{1+\lambda_{k} \rho / K}
$$

Thus, the outage probability (30) becomes

$$
P_{\text {out }}=\prod_{k=1}^{K}\left(1-e^{-2^{R} /\left(1+\lambda_{k} \rho / K\right)}\right), 2^{R} \geq 0 .
$$

However, it is well established in literature 4 that when a signal is transmitted from a spatial region, a finite number of independent channels (modes) are excited at the transmit region to carry information to the receive region. Furthermore, for transmissions between circular regions, the independent channels are represented by Bessel functions [43-45]. While, spherical Bessel functions indicate the the channels for spherical regions $[7,45]$. Thus, to determine the general power policy for communication between circular (or spherical) regions, it is important to study the characteristics of Bessel (and spherical Bessel) functions.

To do so, in Fig. 3, we depict the characteristics curves of Bessel functions and spherical Bessel functions. It is clear from the figure that except for the $0^{\text {th }}$ mode, Bessel (and spherical Bessel) functions start small before increasing monotonically to their maximum. In addition, the Bessel (and spherical Bessel) functions start more slowly as the mode index $n$ increases. Hence, intuitively, the most feasible transmit power allocation scheme for communication between circular (or spherical) regions is to allocate the total transmit power among the independent channels in a descending order.

Based on the above discussion, we propose the following descending transmit power allocation scheme.

\footnotetext{
${ }^{4}$ The works of $[16,27]$ provided a detailed study of the modeling of spatial channels around the transmit and receive regions utilizing modal decomposition techniques.
} 


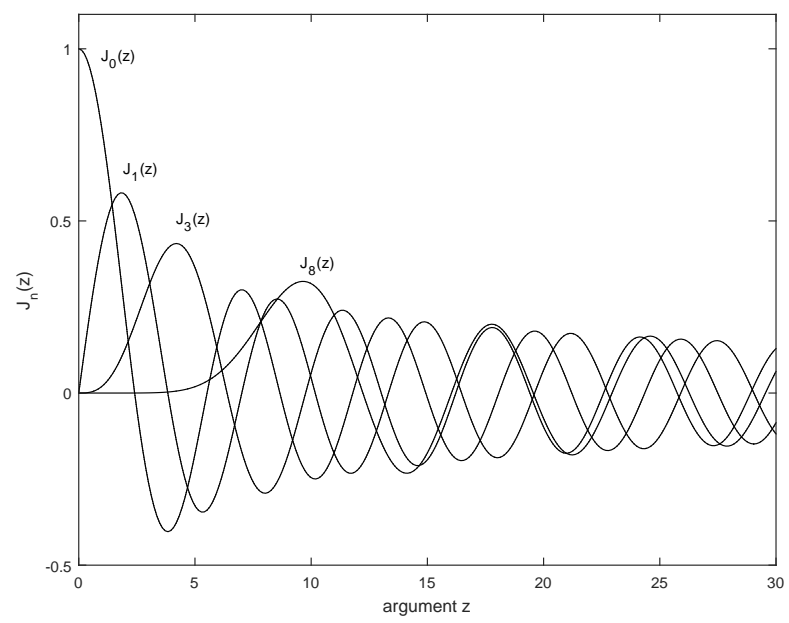

(a) Bessel functions $J_{n}(z)$ vs. argument $z$

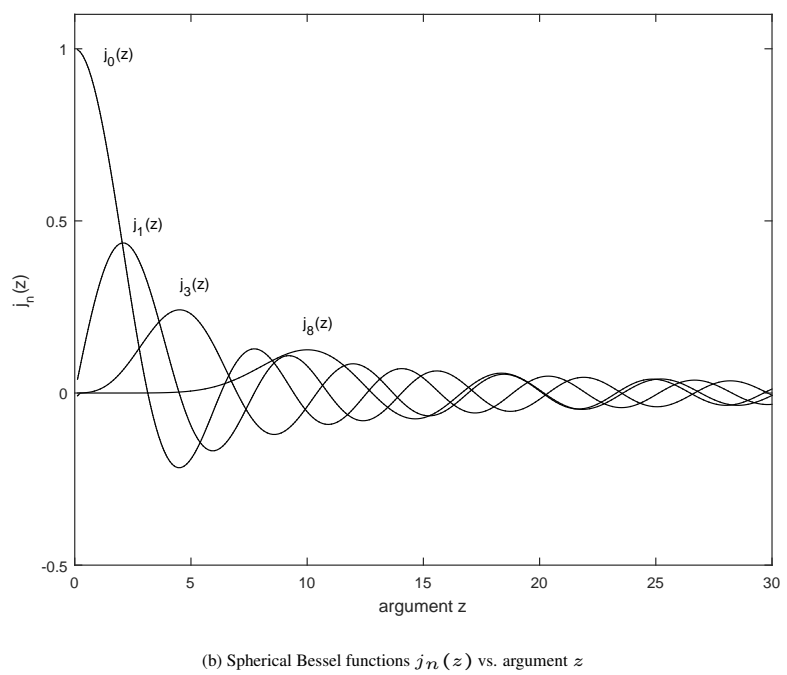

Fig. 3. Characteristics curves of Bessel and Spherical Bessel functions for mode $n=0,1,3,8$ where argument $z$ is a function of frequency and radius of the regions.

Descending Transmit Power Allocation:

In this case, the total transmit power $P_{T}$ is distributed among the $K$ channels in a descending order such that

$$
P_{1}>P_{2}>\cdots>P_{K}
$$

Thus, power at each channel can be represented as

$$
P_{k}=a_{k} P_{T}
$$

where $a_{k}=(0,1)$ such that the following criterions are satisfied

1. $a_{1}>a_{2}>\cdots>a_{K}$.

2. $\sum_{k=1}^{K} a_{k} P_{T} \leq P_{T}$. 
We propose Algorithm 1 to generate $P_{k}$. Here, it should be noted that in this algorithm, due to the exponential property of the spread factor, i.e., $\xi^{k}$, for $\xi \rightarrow 1$, the total transmit power $P_{T}$ is distributed uniformly among the $K$ channels in a descending order. Whereas, for $\xi \leq 0.5$, most of the power is contained in the most favorable $(k=1)$ channel.

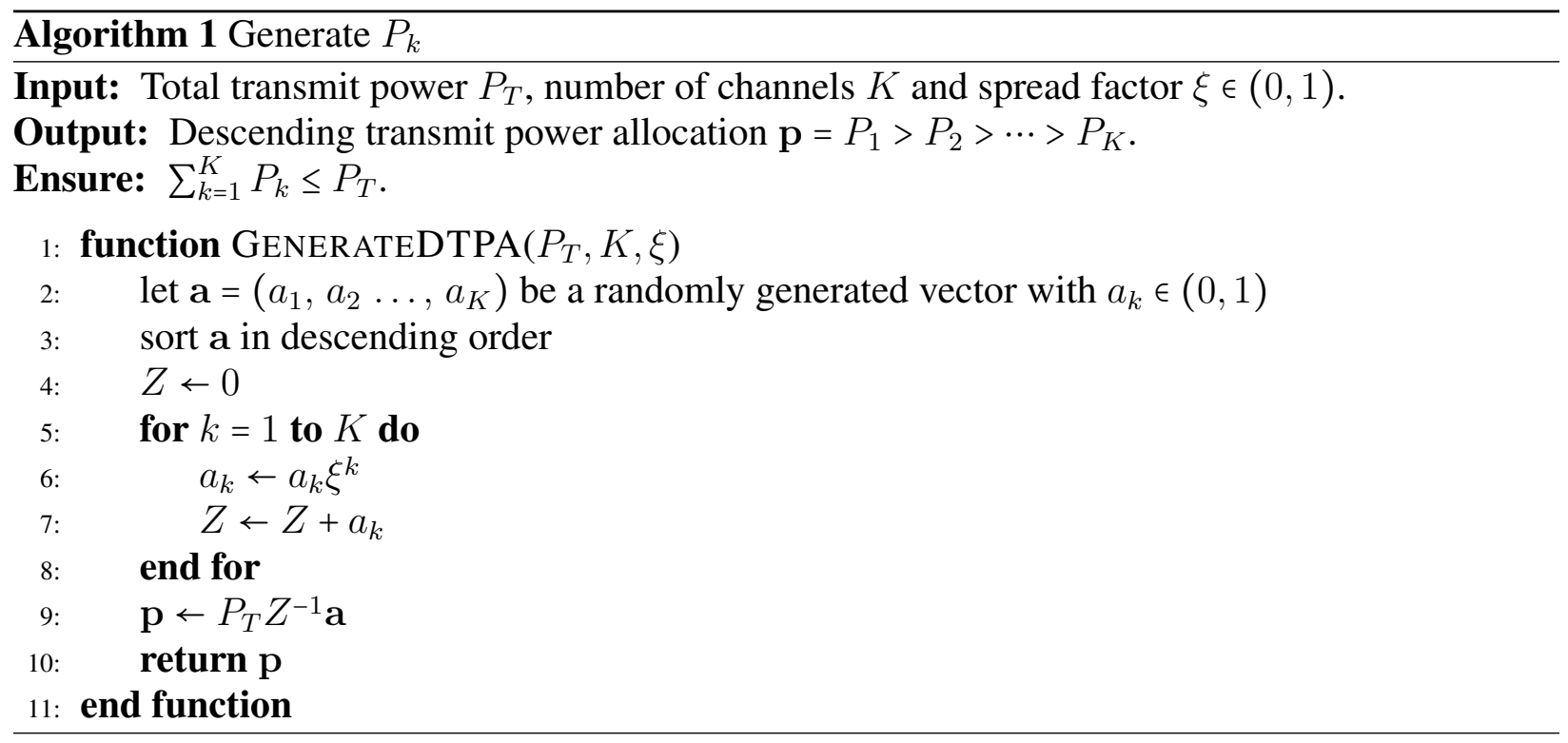

Hence, the instantaneous capacity (28) can be rewritten as

$$
C_{\text {ins }}=\sum_{k=1}^{K} \log \left(1+a_{k} \rho \lambda_{k}\right)
$$

where $\rho$ is the average SNR per receive mode defined in (24).

In addition, the outage probability (30) can be expressed as

$$
P_{\text {out }}=\prod_{k=1}^{K}\left(1-e^{-2^{R} /\left(1+a_{k} \rho \lambda_{k}\right)}\right), 2^{R} \geq 0
$$

where based on (31)

$$
\zeta_{k}=\frac{1}{1+a_{k} \rho \lambda_{k}} .
$$

Note that the proposed algorithm does not give an optimum solution. The optimum power allocation policy would be to water-fill over the eigenvalues $\lambda_{k}$ of the channel matrix. The optimum solution is an interesting open problem.

In the next section, we show numerical comparison between the equal power and descending power allocation schemes.

\section{Numerical Analysis}

In this section, we study the performance of spatially distributed MIMO systems numerically considering a simple Rayleigh fading channel with unit average gain. To do so, we consider circular 
transmit and receive regions of radius $r_{T}=a_{T} \lambda$ and $r_{R}=a_{R} \lambda$, respectively where $\lambda$ represents the wavelength corresponding to the operating frequency and $a_{T}$ and $a_{R}$ are any positive real numbers.

It is evident from the discussion of Section 3.3 that in a rich scattering environment, populating the transmit and the receive regions with different number of antennas (modes) simply adds redundancy to the system. In addition, since the number of effective modes is limited by the size of the regions, the spatially distributed MIMO systems provide best performance when the regions are of same size. Hence, we consider circular transmit and receive regions of radius $r=r_{T}=r_{R}=1 \lambda$. Then according to [43], the effective number of modes in transmit and receive region become $N=M=(2[e \pi r / \lambda\rceil+1)=19$. Further, we exploit Algorithm 1 for different values of spread factor $\xi$.

In Fig. 4, the instantaneous capacity (28) is illustrated as a function of average SNR per receive mode. The figure shows a comparison between the equal power and descending power allocation schemes. It is evident from the figure that

- Considering spread factor $\xi \leq 0.5$ (most of the transmit power is contained in the $k=1$ channel), Fig. 4(a) and Fig. 4(b), the proposed descending power allocation algorithm provides better performance compared to the equal power allocation scheme at low SNR. However, since $k=1$ channel contains most of the transmit power, channels represented with higher values of $k$ have too little power to use. Hence, it is not possible to detect the transmitted signals effectively at high SNR. In such scenarios, the equal transmit power allocation scheme performs better than the proposed scheme. A possible solution to this drawback of the proposed scheme is water-filling over the eigen channels.

- With increasing values of spread factor $\xi$ (total transmit power distributed uniformly among the $K$ channels in a descending order), Fig. 4(c) and Fig. 4(d), the proposed descending power allocation scheme outperforms equal power allocation scheme at both low and high SNR.

The outage probability performance (30) is demonstrated as a function of average SNR per receive mode for different values of spectral efficiency in Fig. 5. This figure provides a comparison between the equal power and descending power allocation schemes for different values of spread factor $\xi$ in Algorithm 1. The figure illustrates that for a desired spectral efficiency, our proposed algorithm outperforms equal power allocation scheme at both low and high SNR. In addition, as can be seen, the smaller values of spread factor $\xi$ provide large performance gains. This finding indicates that the probability of attaining a desired spectral efficiency at a low average SNR is higher when most of the transmit power is contained in the $k=1$ channel, rather than distributing the power uniformly among the $K$ channels in a descending order.

Note that there are two regimes of interest that one can look at for rayleigh fading channel: high SNR and low SNR. While the performance of multi-antenna systems is well studied in high SNR regime, the impact of fading and diversity on capacity is much more significant in low SNR than high SNR. However, determining the performance limits is quite challenging at the low SNR. The simulation results indicate that at low SNR, the proposed algorithm results in significant performance improvement compared to the conventional equal transmit power allocation scheme.

\section{Conclusion}

This work presents a novel framework to represent any spatially distributed MIMO system between arbitrary shaped spatial regions from a mode-to-mode communication perspective. We derive 


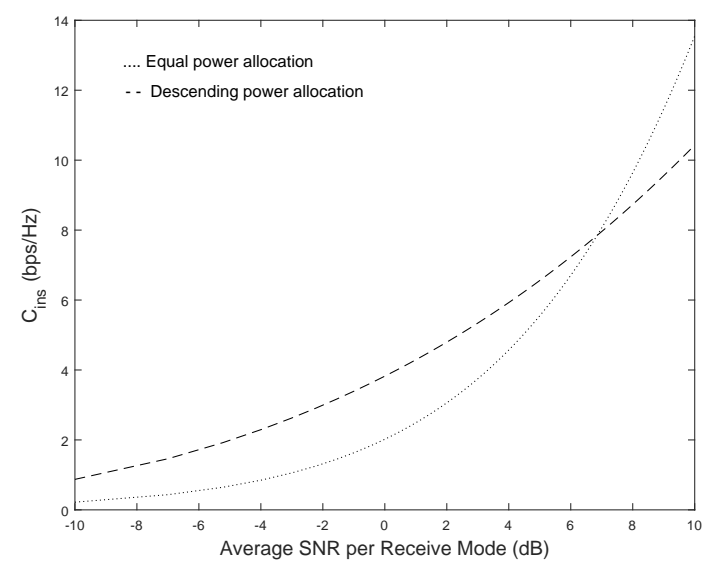

(a) $\xi=0.3$

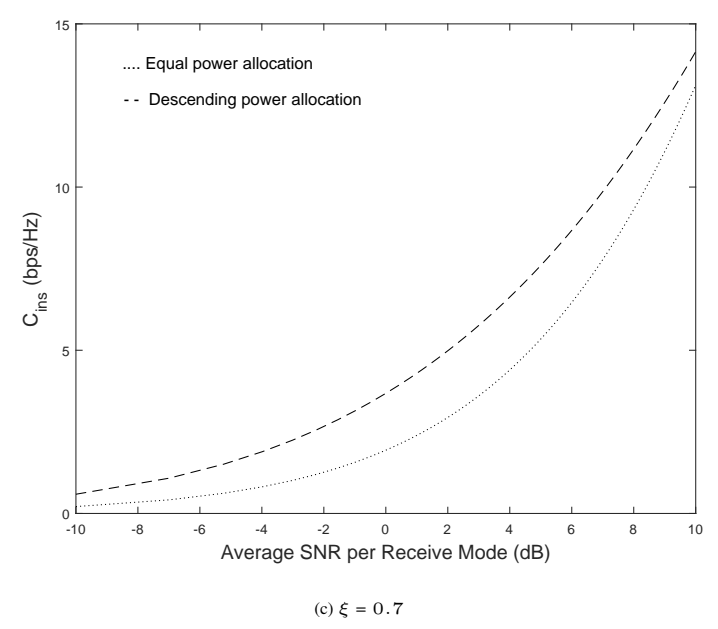

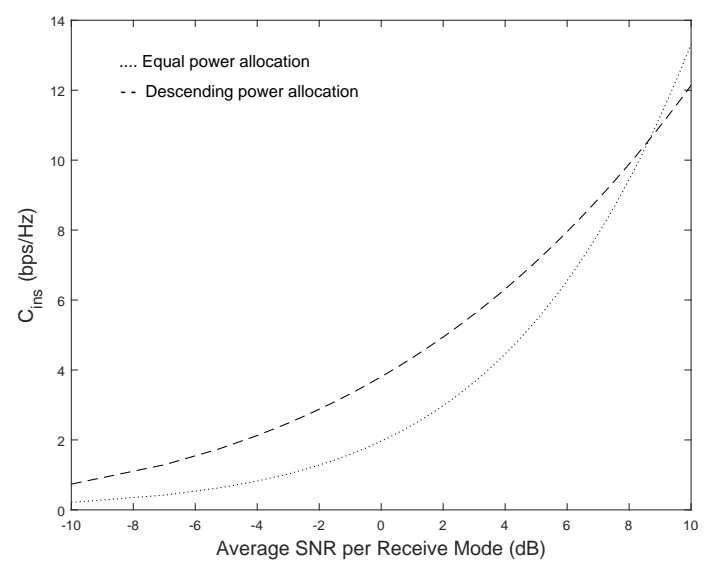

(b) $\xi=0.5$

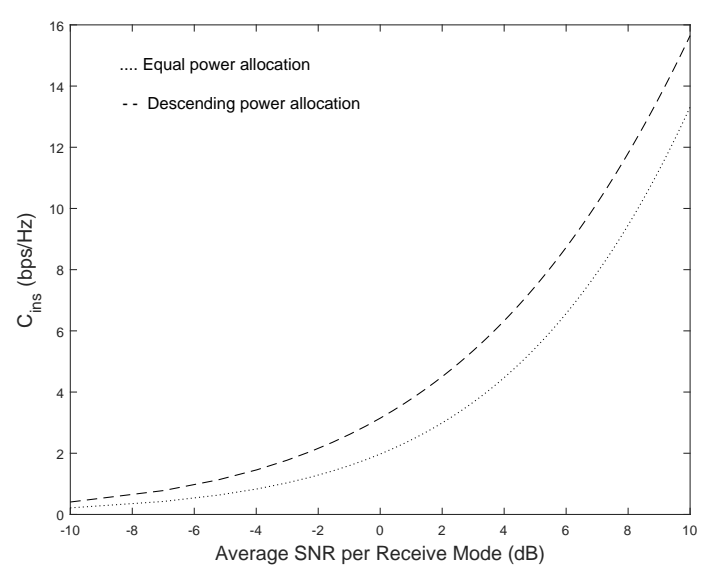

(d) $\xi=0.9$

Fig. 4. Instantaneous capacity as a function of average SNR per receive mode considering different values of spread factor $\xi$ in Algorithm 1.

exact closed-form expressions for the outage probability and instantaneous capacity of spatially distributed MIMO system subject to Rayleigh fading channels. We show that the mathematical formulation to evaluate the performance limits of the proposed system is similar to that of pointto-point MIMO system. Further, in a rich scattering environment, the spatially distributed MIMO system provides best performance when the spatial regions are of same size. Our derived results indicate that analogous to conventional MIMO system, the distributed MIMO system provides best performance with each region containing equal number of randomly distributed antennas, populating regions with different number of antennas simply adds redundancy to the system. In addition, to facilitate the total transmit power allocation among the channels, we look at a descending transmit power allocation algorithm. Simulation results illustrate that the proposed algorithm, where the total transmit power is distributed among the channels in a descending order outperforms the conventional equal power allocation scheme, even at a low SNR. However, the proposed algorithm does not give an optimum solution. An interesting open problem is the development of an optimal power algorithm (e.g. water filling algorithm). 


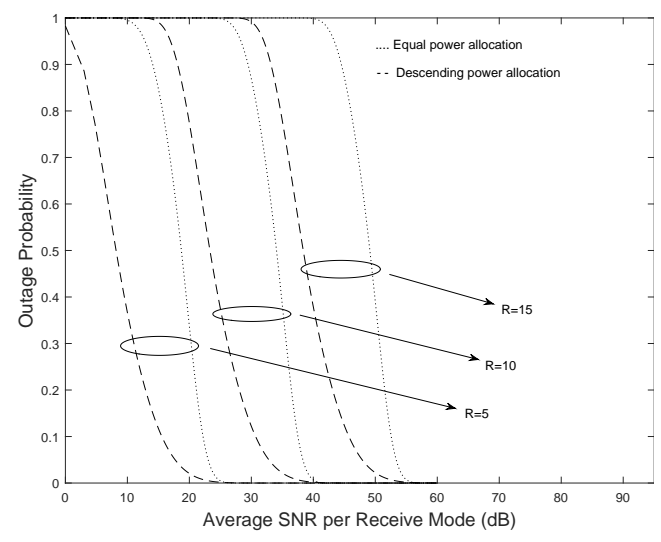

(a) $\xi=0.3$

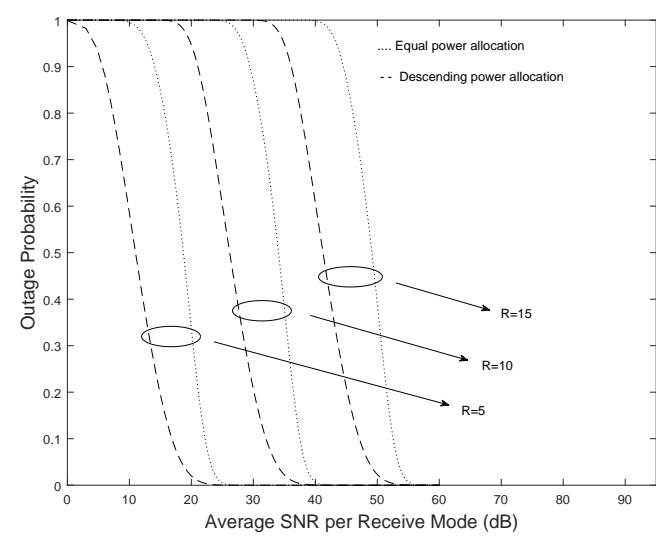

(c) $\xi=0.7$

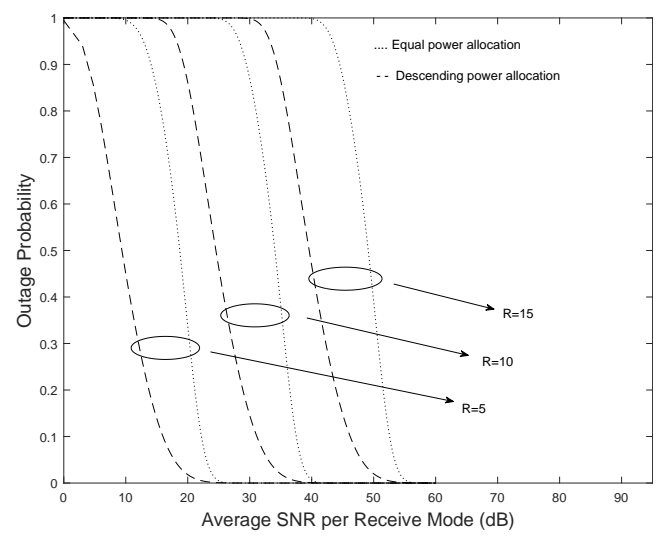

(b) $\xi=0.5$

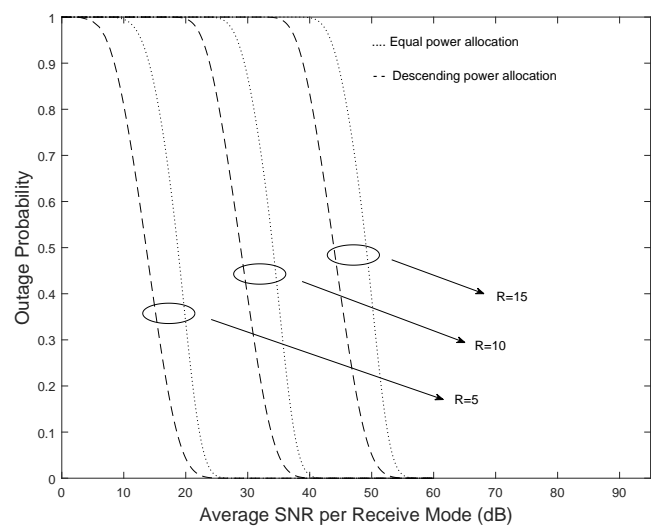

(d) $\xi=0.9$

Fig. 5. Outage probability as a function of average SNR per receive mode for different spectral efficiency $R$ (in bit/s/Hz). We consider different values of spread factor $\xi$ in Algorithm 1.

\section{References}

[1] A. Ozgur, O. Leveque, and D. Tse, "Spatial degrees of freedom of large distributed MIMO systems and wireless ad hoc networks," IEEE Journal on Selected Areas in Communications, vol. 31, no. 2, pp. 202-214, February 2013.

[2] M. Desgroseilliers, O. Leveque, and E. Preissmann, "Spatial degrees of freedom of MIMO systems in line-of-sight environment," in IEEE International Symposium on Information Theory Proceedings, 2013, pp. 834-838.

[3] J. Li, D. Wang, P. Zhu, and X. You, "Spectral efficiency analysis of single-cell multi-user large-scale distributed antenna system," IET Communications, vol. 8, no. 12, pp. 2213-2221, 2014.

[4] — "Spectral efficiency analysis of large-scale distributed antenna system in a composite correlated rayleigh fading channel," IET Communications, vol. 9, no. 5, pp. 681-688, 2015.

[5] P. Gupta and P. R. Kumar, "The capacity of wireless networks," IEEE Transactions on Information Theory, vol. 42, no. 2, pp. 388-404, Mrach 2000. 
[6] D. Gesbert, T. Ekman, and N. Christophersen, "Capacity limits of dense palm-sized MIMO arrays," in IEEE Global Telecommunications Conference, vol. 2, 2002, pp. 1187-1191.

[7] T. D. Abhayapala, T. S. Pollock, and R. Kennedy, "Characterization of 3D spatial wireless channels," in IEEE 58-th Vehicular Technology Conference, VTC 2003-Fall, vol. 1, 2003, pp. $123-127$.

[8] T. Pollock, M. Williams, and T. Abhayapala, "Spatial limits to mutual information scaling in multi-antenna systems," in IEEE International Conference on Acoustics, Speech, and Signal Processing, vol. 3, 2005, pp. 389-392.

[9] L. W. Hanlen and M. Fu, "Wireless communication systems with spatial diversity: a volumetric model," IEEE Transactions On Wireless Communications, vol. 5, no. 1, pp. 133-142, Jan. 2006.

[10] M. Jensen and J. Wallace, "Capacity of the continuous-space electromagnetic channel," IEEE Transactions on Antennas and Propagation, vol. 56, no. 2, pp. 524-531, 2008.

[11] M. Matthaiou, C. Zhong, M. R. McKay, and T. Ratnarajah, "Sum rate analysis of ZF receivers in distributed MIMO systems," IEEE Journal on Selected Areas in Communications, vol. 31, no. 2, pp. 180-191, 2013.

[12] A. Karadimitrakis, A. L. Moustakas, and P. Vivo, "Outage capacity for the optical MIMO channel,” IEEE Transactions on Information Theory, vol. 60, no. 7, pp. 4370-4382, 2014.

[13] M. Kazemi and H. Aghaeinia, "Approximate ergodic capacity of multiuser massive multiple input multiple output in a rayleigh fading uplink channel with variance profile," IET Communications, vol. 9, no. 6, pp. 844-852, 2015.

[14] T. Yang, Q. T. Sun, J. A. Zhang, and J. Yuan, "A linear network coding approach for uplink distributed MIMO systems: Protocol and outage behavior," IEEE Journal on Selected Areas in Communications, vol. 33, no. 2, pp. 250-263, 2015.

[15] D. Fang and A. Burr, "The uplink of distributed MIMO: Wireless network coding versus coordinated multipoint," IEEE Communications Letters, vol. 19, no. 7, pp. 1229-1232, 2015.

[16] T. S. Pollock, "On limits of multi-antenna wireless communications in spatially selective channels," Ph.D. dissertation, The Australian National University, 2003.

[17] F. Bashar and T. D. Abhayapala, "Outage probability analysis for communication between spatial regions," in 10th International Conference on Signal Processing and Communication Systems (ICSPCS2016), 19-21 December 2016, accepted.

[18] D. A. B. Miller, "Spatial channels for communicating with waves between volumes," Optics letters, vol. 23, no. 21, pp. 1645-1647, 1998.

[19] — - "Communicating with waves between volumes: evaluating orthogonal spatial channels and limits on coupling strengths," Applied Optics, vol. 39, no. 11, pp. 1681-1699, Aprill 2000 .

[20] R. Piestun and D. A. B. Miller, "Electromagnetic degrees of freedom of an optical system," Journal of Optical Society of America, vol. 17, no. 5, pp. 892-902, 2000. 
[21] P. J. Smith and M. Shafi, "On a gaussian approximation to the capacity of wireless mimo systems," in IEEE International Conference on Communications (ICC), vol. 1, 2002, pp. 406-410.

[22] A. L. Moustakas, S. H. Simon, and A. M. Sengupta, "Mimo capacity through correlated channels in the presence of correlated interferers and noise: A (not so) large n analysis," IEEE Transactions on Information Theory, vol. 49, no. 10, pp. 2545-2561, 2003.

[23] E. Biglieri and G. Taricco, Transmission and Reception with Multiple Antennas: Theoretical Foundations. Now Publishers Inc, 2004.

[24] B. M. Hochwald, T. L. Marzetta, and V. Tarokh, "Multi-antenna channel-hardening and its implications for feedback and scheduling," IEEE Transaction on Information Theory, vol. 50, no. 9, pp. 1893-1909, 2004.

[25] F. Heliot, R. Hoshyar, and R. Tafazolli, "An accurate closed-form approximation of the distributed MIMO outage probability," IEEE Transactions on Wireless Communications, vol. 10, no. 1, pp. 5-11, 2011.

[26] D. Colton and R. Kress, Inverse acoustic and electromagnetic scattering theory, Applied Mathematical Sciences, 2nd ed. New York: Springer, 1998, vol. 93.

[27] E. G. Williams, Fourier Acoustics: Sound Radiation and Nearfield Acoustical Holography. New York: Academic Press, 1999.

[28] S. Kasturia and J. M. Cioffi, "Vector coding with decision feedback equalization for partial response channels," in IEEE Global Telecommunications Conference, 1988, and Exhibition.' Communications for the Information Age.'Conference Record, GLOBECOM'88. IEEE, 1988, pp. 853-857.

[29] S. Kasturia, J. T. Aslanis, and J. M. Cioffi, "Vector coding for partial response channels," IEEE Transactions on Information Theory, vol. 36, no. 4, pp. 741-762, 1990.

[30] J. M. Cioffi and G D Forney Jr., "Generalized decision-feedback equalization for packet transmission with isi and gaussian noise," in Communications, Computation, Control, and Signal Processing, A Paulraj et al, Ed. Springer, 1997, pp. 79-127.

[31] G. G. Raleigh and J. M. Cioffi, "Spatio-temporal coding for wireless communication," IEEE Transactions on communications, vol. 46, no. 3, pp. 357-366, 1998.

[32] B. Sklar, "Rayleigh fading channels in mobile digital communication systems part i: Characterization," IEEE Communications Magazine, vol. 35, no. 7, pp. 90 -100, July 1997.

[33] D. Gesbert, H. Bölcskei, D. Gore, and A. Paulraj, "MIMO wireless channels: Capacity and performance prediction," in IEEE Global Telecommunications Conference, 2000 (GLOBECOM'00), vol. 2, 2000, pp. 1083-1088.

[34] I. E. Telatar, "Capacity of multi-antenna gaussian channels," European Transactions on Telecommunications, vol. 10, pp. 585-595, 1999.

[35] D. Gesbert, M. Shafi, D. Shiu, P. J. Smith, and A. Naguib, "From theory to practice: An overview of MIMO spacetime coded wireless systems," IEEE Journal On Selected Areas In Communications, vol. 21, no. 3, pp. 281-302, April 2003. 
[36] J. W. Wallace and M. A. Jensen, "Mutual coupling in MIMO wireless systems: A rigorous network theory analysis," IEEE Transactions on Wireless Communications, vol. 3, no. 4, pp. 1317-1325, 2004.

[37] M. Jensen and J. W. Wallace, "A review of antennas and propagation for MIMO wireless communications," IEEE Transactions on Antennas and Propagation, vol. 52, no. 11, pp. 2810-2824, 2004.

[38] Y. Jiang, J. Li, and W. W. Hager, "Joint transceiver design for MIMO communications using geometric mean decomposition," IEEE Transactions on Signal Processing, vol. 53, no. 10, pp. 3791-3803, 2005.

[39] G. Lebrun, J. Gao, and M. Faulkner, "MIMO transmission over a time-varying channel using SVD," IEEE Transactions on Wireless Communications, vol. 4, no. 2, pp. 757-764, 2005.

[40] S. A. Jafar and M. J. Fakhereddin, "Degrees of freedom for the MIMO interference channel," IEEE Transactions on Information Theory, vol. 53, no. 7, pp. 2637-2642, 2007.

[41] S. Shafiee, N. Liu, and S. Ulukus, "Towards the secrecy capacity of the gaussian MIMO wiretap channel: The 2-2-1 channel," IEEE Transactions on Information Theory, vol. 55, no. 9, pp. 4033-4039, 2009.

[42] Y. Liu, Y. Yu, W. Lu, and H. Zhu, "Stochastic multiple-input multiple-output channel model based on singular value decomposition," IET Communications, vol. 9, no. 15, pp. 1852-1856, 2015 .

[43] H. M. Jones, R. A. Kennedy, and T. D. Abhayapala, "On dimensionality of multipath fields: Spatial extent and richness," in IEEE Intl. Conf. Acoustics Speech and Signal Proc., ICASSP, vol. 3, May 2002, pp. 2837-2840.

[44] T. D. Abhayapala, T. S. Pollock, and R. A. Kennedy, "Spatial decomposition of MIMO wireless channels," in Seventh Intl.Symp.on Signal Processing and Its Applications, vol. 1, July 1-4 2003, pp. 309-312.

[45] R. A. Kennedy, P. Sadeghi, T. D. Abhayapala, and H. M. Jones, "Intrinsic limits of dimensionality and richness in random multipath fields," IEEE Transactions on Signal processing, vol. 55, no. 6, pp. 2542-2556, June 2007.

[46] G. J. Foschini, "Layered spacetime architecture for wireless communication in a fading environment when using multielement antennas," Bell Labs Technical Journal, vol. 1, pp. 41-59, 1996.

\section{Appendices}

\section{Appendix 1: Proof of Theorem 1}

Proof. Lets define [34]

$$
\hat{\boldsymbol{H}}= \begin{cases}\boldsymbol{H}^{\dagger} \boldsymbol{H} & M \geq N \\ \boldsymbol{H} \boldsymbol{H}^{\dagger} & M<N\end{cases}
$$


where $\hat{\boldsymbol{H}}$ is an $K \times K$ random non-negative matrix with $K=\min \{N, M\}$ representing the number of independent channels. In effect, the identity matrix and $\boldsymbol{Q}$ in (27) also become $K \times K$ matrices.

However, by singular value decomposition (SVD) theorem, any matrix $\boldsymbol{H} \in \mathcal{C}^{M \times N}$ in (27) can be written as $\boldsymbol{H}=\boldsymbol{U} \boldsymbol{D} \boldsymbol{V}^{\dagger}$, where $\boldsymbol{U} \in \mathcal{C}^{M \times M}$ and $\boldsymbol{V} \in \mathcal{C}^{N \times N}$ are unitary, and $\boldsymbol{D} \in \mathcal{R}^{M \times N}$ is non-negative define and diagonal. Further, $\boldsymbol{D}=\operatorname{diag}\left\{\sqrt{\lambda_{1}}, \sqrt{\lambda_{2}}, \ldots \sqrt{\lambda_{K}}, 0, \cdots 0\right\}$, where $\sqrt{\lambda_{k}}$, $k=\{1,2, \ldots K\}$ are the singular values of the channel matrix.

Consider that

$$
\hat{\boldsymbol{Q}}= \begin{cases}\boldsymbol{V}^{\dagger} \boldsymbol{Q} \boldsymbol{V} & M \geq N \\ \boldsymbol{U}^{\dagger} \boldsymbol{Q} \boldsymbol{U} & M<N\end{cases}
$$

and

$$
\boldsymbol{\Lambda}= \begin{cases}\boldsymbol{D}^{\dagger} \boldsymbol{D} & M \geq N \\ \boldsymbol{D} \boldsymbol{D}^{\dagger} & M<N\end{cases}
$$

Then we can rewrite (27) as

$$
\mathcal{I}(s, \boldsymbol{y} \mid \boldsymbol{H})=\log \left|\boldsymbol{I}_{K}+\frac{1}{\sigma^{2}} \boldsymbol{\Lambda} \hat{\boldsymbol{Q}}\right|
$$

where $\operatorname{tr}(\hat{\boldsymbol{Q}})=\operatorname{tr}(\boldsymbol{Q})$. Observe that to maximize (44), $\hat{\boldsymbol{Q}}$ has to be a diagonal matrix, $\hat{\boldsymbol{Q}}=$ $\operatorname{diag}\left\{P_{1}, P_{2}, \cdots P_{K}\right\}$ with optimal power distribution $P_{k}$ such that

$$
\sum_{k=1}^{K} P_{k} \leq P_{T}
$$

If, however, $\lambda_{k}$ is the $(k, k)^{t h}$ element of $\Lambda$, then $\lambda_{k}$ represents the $k^{\text {th }}$ eigenvalue of $\hat{\boldsymbol{H}}$ (41).

Based on this mathematical formalism, the instantaneous capacity of the proposed spatially distributed MIMO system (27) can be rewritten as (28)

\section{Appendix 2: Proof of Theorem 2}

Proof. Observed that based on Theorem 1, $\mathcal{I}(\boldsymbol{s}, \boldsymbol{y} \mid \boldsymbol{H})$ in (29) can be rewritten as

$$
\begin{aligned}
\mathcal{I}(s, \boldsymbol{y} \mid \boldsymbol{H}) & =C_{\text {ins }} \\
& =\sum_{k=1}^{K} \log \left(1+\frac{1}{\sigma^{2}} P_{k} \lambda_{k}\right) \\
& =\log \left[\left(1+\frac{P_{1}}{\sigma^{2}} \lambda_{1}\right)\left(1+\frac{P_{2}}{\sigma^{2}} \lambda_{2}\right) \cdots\left(1+\frac{P_{K}}{\sigma^{2}} \lambda_{K}\right)\right] \\
& =\log \left(\prod_{k=1}^{K}\left(1+\frac{P_{k}}{\sigma^{2}} \lambda_{k}\right)\right) .
\end{aligned}
$$

In effect, (29) can be expressed as follows

$$
P_{\text {out }}=\operatorname{Pr}\left\{\prod_{k=1}^{K}\left(1+\frac{P_{k}}{\sigma^{2}} \lambda_{k}\right)<2^{R}\right\} .
$$


Lets denote $w_{k}=\left(1+\frac{P_{k}}{\sigma^{2}} \lambda_{k}\right)$. Now, since we consider Rayleigh fading, $w_{k} \mathrm{~s}$ ' can be thought of as exponentially distributed random variables with rate $\zeta_{k}$ where $\zeta_{k}$ is defined in (31). Hence, the cdf of $w_{k}$ for $k=1,2, \cdots K$ is given by

$$
\operatorname{Pr}\left\{w_{k}<w\right\}=1-e^{-w \zeta_{k}}, w \geq 0 .
$$

Now, define a random variable $W=\prod_{k=1}^{K} w_{k}$. Considering the $\zeta_{k} \mathrm{~s}^{\prime}$ are distinct, the cdf of $W$ can be given as

$$
\operatorname{Pr}\{W<w\}=\prod_{k=1}^{K}\left(1-e^{-w \zeta_{k}}\right), \quad w \geq 0 .
$$

Thus, the outage probability can be expressed in terms of the cdf of $W$ as

$$
P_{\text {out }}=\operatorname{Pr}\left\{W<2^{R}\right\} \text {. }
$$

Note that using (49) in (50) and replacing $W=\prod_{k=1}^{K} w_{k}$ where $w_{k}=\left(1+\lambda_{k} P_{k} / \sigma^{2}\right)$, we obtain Theorem 2. 